\title{
Dissipation and Rheology of Sheared Soft-Core Frictionless Disks Below Jamming
}

\author{
Daniel Vågberg, ${ }^{1}$ Peter Olsson, ${ }^{1}$ and S. Teitel ${ }^{2}$ \\ ${ }^{1}$ Department of Physics, Umeå University, 90187 Umeå, Sweden \\ ${ }^{2}$ Department of Physics and Astronomy, University of Rochester, Rochester, New York 14627, USA
}

(Received 19 November 2013; published 20 May 2014)

\begin{abstract}
We use numerical simulations to investigate the effect that different models of energy dissipation have on the rheology of soft-core frictionless disks, below jamming in two dimensions. We find that it is not necessarily the mass of the particles that determines whether a system has Bagnoldian or Newtonian rheology, but rather the presence or absence of large connected clusters of particles. We demonstrate the key role that tangential dissipation plays in the formation of such clusters and in several models find a transition from Bagnoldian to Newtonian rheology as the packing fraction $\phi$ is varied. For each model, we show that appropriately scaled rheology curves approach a well defined limit as the mass of the particles decreases and collisions become strongly inelastic.
\end{abstract}

DOI: 10.1103/PhysRevLett.112.208303

PACS numbers: 83.80.Iz, 83.60.Rs, 83.80.Fg

Many seemingly disparate physical systems, such as granular materials, foams, emulsions, and suspensions, have been modeled in terms of soft-core interacting particles in an athermal limit. Such a common description has led to the prediction of common physical behaviors, notably the jamming transition from a liquidlike state to a rigid but disordered solid [1-3]. Of particular interest has been the behavior of such systems under a steady shear strain rate $\dot{\gamma}$ [4-7]. Below jamming, granular particles are usually described by Bagnoldian rheology [8-11] with pressure $p$ and shear stress $\sigma$ scaling $\propto \dot{\gamma}^{2}$ at low $\dot{\gamma}$. However, foams and emulsions are found to obey Newtonian rheology [7,12-14] with $p, \sigma \propto \dot{\gamma}$ at low $\dot{\gamma}$. It is, therefore, important to understand what are the essential features of the microscopic interactions that lead to one rheology or the other.

Here, we consider within a unified framework the effect that different, commonly used, models of energy dissipation have on the rheology of soft-core frictionless disks, below jamming in two dimensions (2D). We find that it is not necessarily the mass of the particles that determines whether a system has Bagnoldian or Newtonian rheology but rather the absence or presence of large connected clusters of particles. For Bagnoldian rheology we find, even in the dense limit, that as $\dot{\gamma} \rightarrow 0$ the average contact number $z \rightarrow 0$ and there are no instantaneous force chains. In contrast, Newtonian rheology requires the formation of large connected clusters of particles, with extended force chains as jamming is approached. We demonstrate the key role that tangential dissipation plays in the formation of such clusters and in several models find a sharp transition from Bagnoldian to Newtonian rheology as the packing fraction $\phi$ is varied. For each model, we show that appropriately scaled rheology curves approach a well defined limit as the mass of the particles decreases and collisions become strongly inelastic.
Our soft-core model is as follows. We take the elastic force on a particle at position $\mathbf{r}_{i}$ due to its contact with a particle at $\mathbf{r}_{j}$ to be

$$
\mathbf{f}_{i j}^{\mathrm{el}}=-k_{e} \frac{d V\left(\left|\mathbf{r}_{i j}\right| / d_{i j}\right)}{d \mathbf{r}_{i}}, \quad \mathbf{r}_{i j} \equiv \mathbf{r}_{i}-\mathbf{r}_{j}
$$

Here, $d_{i j}=\left(d_{i}+d_{j}\right) / 2$ is the average diameter of the two particles, $V(x)$ is a dimensionless soft-core interaction potential with $V(x)=0$ for $x>1$, and $k_{e}$ is the coupling that sets the energy scale of the interaction.

For the dissipative force, we consider several different models. In the "reservoir dissipation" (RD) model, a particle with center of mass velocity $\mathbf{v}_{i}=\dot{\mathbf{r}}_{i}$ decays to the average shear flow velocity. For uniform shear flow in the $x$ direction, we have

$$
\mathbf{f}_{i}^{\text {dis }}=-k_{d}\left[\mathbf{v}_{i}-\dot{\gamma} y_{i} \hat{\mathbf{x}}\right] \quad \text { for model RD. }
$$

In the "contact dissipation" (CD) model, we assume dissipation is due to binary collisions between particles. We take the force on particle $i$ due to contact with particle $j$ to be

$$
\mathbf{f}_{i j}^{\mathrm{dis}}=-k_{d}\left[\mathbf{v}_{i}-\mathbf{v}_{j}\right] \text { for model CD. }
$$

We also consider the model $\mathrm{CD}_{n}$ in which dissipation is due only to the velocity difference in the direction normal to the point of contact,

$$
\mathbf{f}_{i j}^{\mathrm{dis}}=-k_{d}\left[\left(\mathbf{v}_{i}-\mathbf{v}_{j}\right) \cdot \hat{r}_{i j}\right] \hat{r}_{i j} \text { for model } \mathrm{CD}_{n} .
$$

For theoretical completeness, we also consider the model $\mathrm{CD}_{t}$, 


$$
\mathbf{f}_{i j}^{\mathrm{dis}}=-k_{d}\left[\left(\mathbf{v}_{i}-\mathbf{v}_{j}\right) \cdot \hat{\mathbf{t}}_{i j}\right] \hat{\mathbf{t}}_{i j} \text { for model } \mathrm{CD}_{t},
$$

where $\hat{\mathbf{t}}_{i j} \equiv \hat{\mathbf{z}} \times \hat{\mathbf{r}}_{i j}$ is tangent to the point of contact.

$\mathrm{CD}_{n}$ is typically used in models of massive dry granular particles [15]. CD was introduced by Durian [12] to describe the viscous interaction between massless foam bubbles. In that context, Durian also introduced RD as a mean-field approximation to $\mathrm{CD}[12,16]$, in which the instantaneous velocity $\mathbf{v}_{j}$ is replaced by its ensemble average $\dot{\gamma} y_{j} \hat{\mathbf{x}}$. However, RD can also be considered as a model for particles embedded in a uniformly sheared host medium, where dissipation is between the particles and the degrees of freedom that comprise the host, such as the Stokes drag on a particle in a fluid. Both CD and RD may be used to model massive particles in emulsions and suspensions [17].

To model a uniform shear flow, we use Lees-Edwards boundary conditions [18]. It is convenient to define the "lab frame" coordinates $\mathbf{r}_{i}$ in terms of "shear frame" coordinates $\left(x_{i}, y_{i}\right)$ that obey periodic boundary conditions $\mathbf{r}_{i} \equiv\left(x_{i}+\gamma y_{i}, y_{i}\right)$, where $\gamma=\dot{\gamma} t$ is the total shear strain in time $t$. The equations of motion for a particle of mass $m_{i}$ can then be written as

$$
m_{i}\left(\ddot{x}_{i}+2 \dot{\gamma} \dot{y}_{i}\right)=\left(f_{i x}-\gamma f_{i y}\right), \quad m_{i} \ddot{y}_{i}=f_{i y},
$$

where $\mathbf{f}_{i}=\mathbf{f}_{i}^{\mathrm{el}}+\mathbf{f}_{i}^{\mathrm{dis}}$ is the total force on the particle.

Generalizing the work of Lemaitre et al. [19], who considered only hard-core particles, we now cast our equation of motion into dimensionless form. We take $d_{s}$ and $m_{s}$, the diameter and mass of our small particles, as our unit of length and mass and $1 / \dot{\gamma}$ as our unit of time. Important time scales in the problem are the elastic and dissipative relaxation times

$$
\tau_{e} \equiv \sqrt{m_{s} d_{s}^{2} / k_{e}}, \quad \tau_{d} \equiv m_{s} / k_{d}
$$

as well as the time

$$
\tau_{0} \equiv \tau_{e}^{2} / \tau_{d}=k_{d} d_{s}^{2} / k_{e},
$$

which is independent of the mass scale $m_{s}$. The degree of elasticity of collisions is conveniently expressed in terms of the ratio

$$
Q \equiv \tau_{d} / \tau_{e}=\sqrt{m_{s} k_{e} /\left(k_{d}^{2} d_{s}^{2}\right)} \propto \sqrt{m_{s}} .
$$

For a harmonic elastic interaction, a head-on collision will be totally inelastic (coefficient of restitution $e=0$ ) when $Q<\frac{1}{2}\left[m_{s} d_{i j}^{2} /\left(\bar{m} d_{s}^{2}\right)\right]^{1 / 2}$, where $\bar{m}$ is the reduced mass of the colliding particles [15].

The equations of motion for the dimensionless trajectories $\left\{X_{i}(\gamma), Y_{i}(\gamma)\right\} \equiv\left\{x_{i}(t) / d_{s}, y_{i}(t) / d_{s}\right\}$ are then

$$
\begin{aligned}
\rho_{i}\left(\dot{\gamma} \tau_{e}\right)^{2}\left[X_{i}^{\prime \prime}+2 Y_{i}^{\prime}\right] & =\left[F_{i x}^{\mathrm{el}}-\gamma F_{i y}^{\mathrm{el}}\right]+\dot{\gamma} \tau_{0}\left[F_{i x}^{\mathrm{dis}}-\gamma F_{i y}^{\mathrm{dis}}\right], \\
\rho_{i}\left(\dot{\gamma} \tau_{e}\right)^{2} Y_{i}^{\prime \prime} & =F_{i y}^{\mathrm{el}}+\dot{\gamma} \tau_{0} F_{i y}^{\mathrm{dis}},
\end{aligned}
$$

where $\rho_{i} \equiv m_{i} / m_{s}, \quad X_{i}^{\prime} \equiv d X_{i} / d \gamma$. The dimensionless forces are

$\mathbf{F}_{i}^{\mathrm{el}}\left(\left\{\mathbf{R}_{i}\right\}\right) \equiv\left(d_{s} / k_{e}\right) \mathbf{f}_{i}^{\mathrm{el}}\left(\left\{\mathbf{r}_{i}\right\}\right)$,

$\mathbf{F}_{i}^{\mathrm{dis}}\left(\left\{\mathbf{R}_{i}^{\prime}\right\}\right) \equiv\left(1 / k_{d} d_{s} \dot{\gamma}\right) \mathbf{f}_{i}^{\mathrm{dis}}\left(\left\{\mathbf{v}_{i}\right\}\right)$,

where the dimensionless lab frame trajectories $\mathbf{R}_{i} \equiv\left(X_{i}+\right.$ $\left.\gamma Y_{i}, Y_{i}\right)$ depend on the strain rate only though the dimensionless strain parameters $\dot{\gamma} \tau_{e}$ and $\dot{\gamma} \tau_{0}=\dot{\gamma} \tau_{e} / Q$.

To study the rheology, we are interested in the stress tensor. Here, we consider only the elastic part $\mathbf{p}^{\mathrm{el}}$, which dominates over the kinetic and dissipative parts for all but the largest $\dot{\gamma}$. Since $\mathbf{p}^{\mathrm{el}} \equiv L^{-D} \sum_{i<j} \mathbf{f}_{i j}^{\mathrm{el}} \otimes \mathbf{r}_{i j}$ in $D$ dimensions [20], we can define a dimensionless stress tensor

$$
\mathbf{P}^{\mathrm{el}} \equiv\left(\frac{d_{s}}{L}\right)^{D} \sum_{i<j} \mathbf{F}_{i j}^{\mathrm{el}} \otimes \mathbf{R}_{i j}=\frac{\tau_{e}^{2} d_{s}^{D-2}}{m_{s}} \mathbf{p}^{\mathrm{e}},
$$

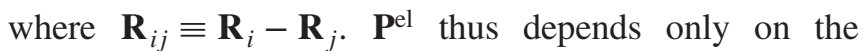
dimensionless trajectories $\mathbf{R}_{i}(\gamma)$, and so plotting the pressure $P^{\mathrm{el}}=\frac{1}{2} \operatorname{tr}\left[\mathbf{P}^{\mathrm{el}}\right]$ vs $\dot{\gamma} \tau_{e}$, all models with the same $Q$ value will fall on the same curve, independent of the specific values of $k_{e}, k_{d}$, and $m_{s}$. In particular, the hard-core limit $k_{e} \rightarrow \infty$, with $k_{d} \sim \sqrt{k_{e}}$ so that $Q$ stays constant, will also lie on the same curve. Since $\tau_{e} \rightarrow 0$ in this limit, we conclude that the hard-core limit may be inferred from softcore simulations, provided one looks at sufficiently small $\dot{\gamma}$.

In our simulations of the above four dissipative models, we have observed two limiting forms of behavior: (i) the "overdamped" limit, where the kinetic term is negligible and the trajectories $\mathbf{R}_{i}(\gamma)$ are determined by the balance of elastic and dissipative terms-here one has Newtonian rheology at small $\dot{\gamma}$; and (ii) the "inertial" limit, where the dissipative term is negligible and the trajectories are determined by the balance of elastic and kinetic termshere one has Bagnoldian rheology at small $\dot{\gamma}$.

(i) The overdamped limit results when the kinetic term in Eq. (10) becomes negligible. We then have

$$
\mathbf{F}_{i}^{\mathrm{el}}=-\left(\dot{\gamma} \tau_{0}\right) \mathbf{F}_{i}^{\mathrm{dis}},
$$

and the dimensionless trajectories, thus, depend parametrically only on the parameter $\dot{\gamma} \tau_{0}$. Assuming the trajectories have a well defined limit as $\dot{\gamma} \rightarrow 0$, then the leading dependence of $\mathbf{F}_{i}^{\mathrm{el}}$ on $\dot{\gamma}$, and so presumably the pairwise contact forces $\mathbf{F}_{i j}^{\mathrm{el}}$ and so also the stress tensor $\mathbf{P}^{\mathrm{el}}$, is $\propto \dot{\gamma} \tau_{0}$, and so one has Newtonian scaling. Deviations from Newtonian scaling will occur at larger $\dot{\gamma}$ due to soft-core effects; these become stronger and set in at lower $\dot{\gamma}$ as one approaches the jamming $\phi_{J}$. But the characteristic feature of the overdamped limit is that curves of $P^{\mathrm{el}}$, when plotted 
vs $\dot{\gamma} \tau_{0}$, will approach a common limiting curve for different $Q$ values. The dimensionless $\tilde{\eta}_{p} \equiv P^{\mathrm{el}} /\left(\dot{\gamma} \tau_{0}\right)$ approaches a constant as $\dot{\gamma} \tau_{0} \rightarrow 0$, giving the hard-core limit. The pressure analog of viscosity is then $p / \dot{\gamma}=k_{d} \tilde{\eta}_{p}$.

(ii) The inertial limit results when the dissipative term becomes negligible. Equation (10) then becomes

$F_{i x}^{\mathrm{el}}=\rho_{i}\left(\dot{\gamma} \tau_{e}\right)^{2}\left[X_{i}^{\prime \prime}(\gamma)+2 Y_{i}^{\prime}(\gamma)+\gamma Y_{i}^{\prime \prime}(\gamma)\right]$,

$F_{i y}^{\mathrm{el}}=\rho_{i}\left(\dot{\gamma} \tau_{e}\right)^{2} Y_{i}^{\prime \prime}(\gamma)$,

and the dimensionless trajectories now depend parametrically only on the parameter $\dot{\gamma} \tau_{e}$. The leading dependence of $\mathbf{F}_{i}^{\mathrm{el}}$ on $\dot{\gamma}$ and so the stress tensor $\mathbf{P}^{\mathrm{el}}$ is $\propto\left(\dot{\gamma} \tau_{e}\right)^{2}$. One, thus, has Bagnold scaling. Deviations from Bagnold scaling will occur at larger $\dot{\gamma}$ due to soft-core effects; these become stronger and set in at lower $\dot{\gamma}$ as one approaches the jamming $\phi_{J}$. But the characteristic feature of the inertial limit is that curves of $P^{\mathrm{el}}$, when plotted vs $\dot{\gamma} \tau_{e}$, will approach a common limiting curve for different $Q$. The dimensionless $B_{p} \equiv P^{\mathrm{el}} /\left(\dot{\gamma} \tau_{e}\right)^{2}$ approaches a constant as $\dot{\gamma} \tau_{e} \rightarrow 0$, giving the hard-core limit. The Bagnold coefficient for pressure is then $p^{\mathrm{el}} / \dot{\gamma}^{2}=m_{s} B_{p}$.

We now present the results of our numerical simulations. Our simulations are for $N=1024$ total disks in $2 \mathrm{D}$, with equal numbers of big and small particles with diameter ratio $d_{b} / d_{s}=1.4$ and $d_{s}=1$ [20]. Finite size effects are negligible for the range of parameters studied here. Although the particles are of different size, we take them to have equal mass $m_{b}=m_{s} \equiv m$. We simulate at fixed packing fraction $\phi=\left(\pi N / 8 L^{2}\right)\left(d_{s}^{2}+d_{b}^{2}\right)$, where the system area $L^{2}$ is varied to achieve the desired $\phi$ value. We use a harmonic interaction $V(x)=\frac{1}{2}(1-x)^{2}$ for $x<1$, with fixed elastic coupling $k_{e}=1$, and vary $k_{d}$ and $m_{s}$ to get different values of $Q$. We integrate the equations of motion (10) using a modified velocity-Verlet algorithm with a Heun-like prestep to account for the velocity-dependent acceleration. We shear to a total strain $\gamma \sim 0.5-50$, depending on system parameters, collecting data only after the system appears to be in steady state. Our main results for the four different dissipative models are presented in Fig. 1, where we plot the dimensionless pressure $P^{\mathrm{el}}$ vs the dimensionless strain rate, $\dot{\gamma} \tau_{e}$ or $\dot{\gamma} \tau_{0}$ (chosen according to the behavior we find in each particular model), for a wide range of $Q$. We show results for a dilute case $\phi=0.60$ as well as for a dense case $\phi=0.82$ just below the jamming $\phi_{J} \approx 0.843$.

For model RD, Figs. 1(a) and 1(b) show Newtonian rheology at small $\dot{\gamma}$. At small $Q \lesssim 2$, we see the overdamped limit, with all data approaching a common limiting curve over 9 orders of magnitude in $\dot{\gamma} \tau_{0}$, spanning the range from Newtonian behavior at small $\dot{\gamma} \tau_{0}$ to non-Newtonian soft-core behavior, with accompanying shear thinning (slope $<1$ ), as $\dot{\gamma} \tau_{0}$ increases; as $\phi$ increases, the onset of this shear thinning moves to lower values of $\dot{\gamma} \tau_{0}$ as expected. For larger $Q$, the curves approach the common
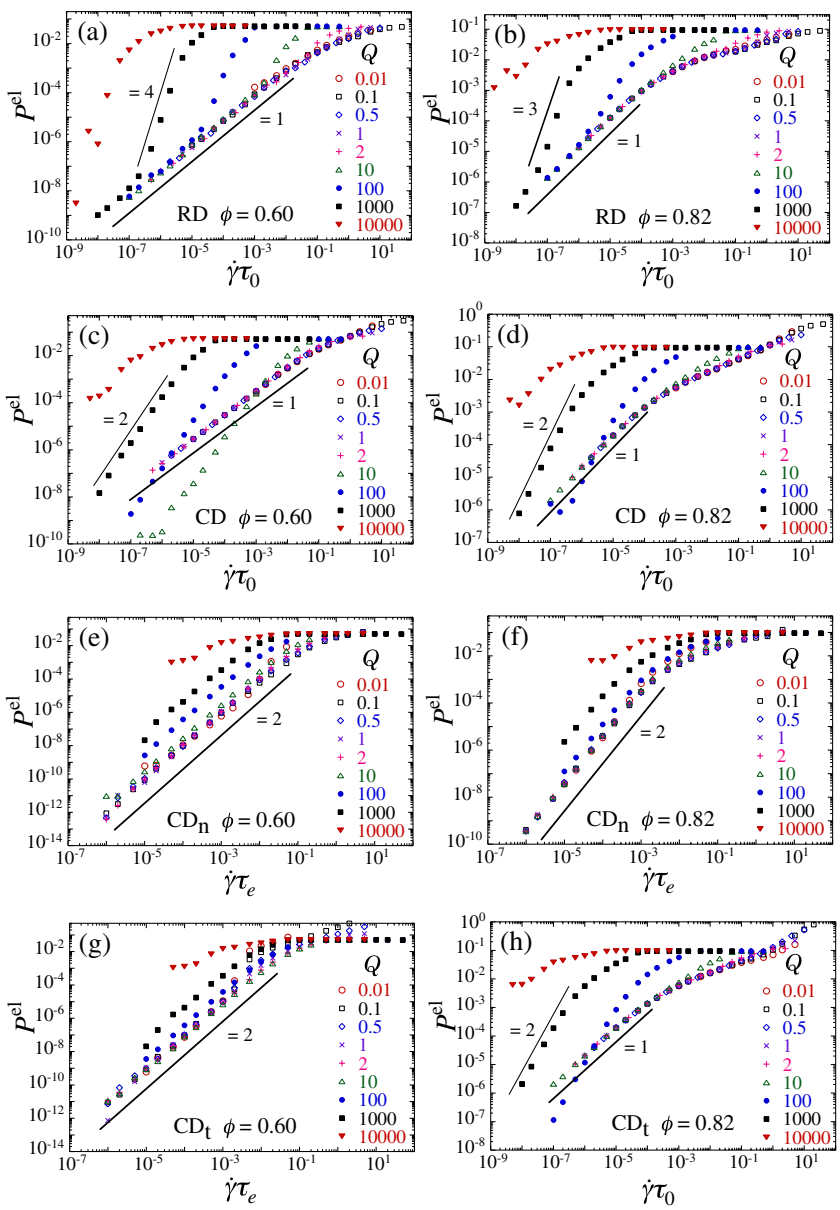

FIG. 1 (color online). Dimensionless pressure $P^{\mathrm{el}}$ vs dimensionless strain rate $\dot{\gamma} \tau_{0}$ or $\dot{\gamma} \tau_{e}$ for different values of $Q$ for the four dissipative models of Eqs. (2)-(5). Left hand column is for packing fraction $\phi=0.60$; right hand column is for $\phi=0.82$, close below jamming. For each value of $Q$, several different choices of $m_{s}$ and $k_{d}$ were used. Straight lines indicate algebraic behaviors, with power law as indicated by the neighboring number.

limiting curve as $\dot{\gamma} \tau_{0} \rightarrow 0$. This is to be expected when the dimensionless $\mathbf{F}_{i}^{\text {dis }}$ in Eq. (10) is at least as big as the dimensionless kinetic factor $\mathbf{R}_{i}^{\prime \prime}$; then the kinetic term on the left hand side of Eq. (10) becomes negligible compared to the dissipative term on the right hand side, as $\dot{\gamma} \tau_{0} \rightarrow 0$ for any $Q$; one thus gets the overdamped limit. This suggests that for RD the hard-core limit of $\tilde{\eta}_{p}$ is independent of $Q$ and, hence, of the mass $m_{s}$. However, as $\dot{\gamma} \tau_{0}$ increases, we see the onset of shear thickening (slope $>1$ ) due to inertial effects, as has been reported previously [21]. As $Q$ increases at fixed $\phi$, this shear thickening onset moves to lower $\dot{\gamma} \tau_{0}$; at fixed $Q$ it moves to lower $\dot{\gamma} \tau_{0}$ as $\phi$ increases. The saturation of $P^{\mathrm{el}}$ at large $\dot{\gamma} \tau_{0}$ represents the limit where particles have so much kinetic energy that the soft-core particles are able to pass through each other.

For model CD, shown in Figs. 1(c) and 1(d), behavior at low $Q$ appears qualitatively similar to that of $\mathrm{RD}$; we are in 
the overdamped limit. In a separate work [22], we will argue that the criticality of the jamming transition for $\mathrm{CD}$ is the same as for $\mathrm{RD}$ as $Q \rightarrow 0$. However, as $Q$ increases we see a transition at $Q^{*}$ from Newtonian $(\sim \dot{\gamma})$ to Bagnoldian $\left(\sim \dot{\gamma}^{2}\right)$ rheology [23]. Comparing Fig. 1(c) with Fig. 1(d), we see that $Q^{*}$ increases with increasing $\phi$.

For model $\mathrm{CD}_{n}$, Figs. 1(e) and 1(f) show Bagnold rheology at small $\dot{\gamma}$ for all values of $Q$. At low $\dot{\gamma} \tau_{e}$, we see shear thickening, with a slope $\sim 2>1$, but as $\dot{\gamma} \tau_{e}$ increases, we see a crossover to shear thinning (slope $<1$ ) due to soft-core effects. As $\phi$ increases, this departure from Bagnold rheology moves to lower values of $\dot{\gamma} \tau_{e}$ as expected. In both Figs. 1(e) and 1(f), we see that the inertial limit holds, with the data approaching a common limiting curve over 7 orders of magnitude in $\dot{\gamma} \tau_{e}$, for a range of small $Q \lesssim 2$, extending to larger $Q$ as $\phi$ increases. However, while our smallest $Q=0.01$ value agrees with this limiting curve at small $\dot{\gamma} \tau_{e}$, it shows a clear departure increasing towards larger values of $P^{\mathrm{el}}$ as $\dot{\gamma} \tau_{e}$ increases.

Finally, our results for $\mathrm{CD}_{t}$ are shown in Figs. 1(g) and 1(h). Here we find Bagnoldian rheology and the inertial limit, similar to model $\mathrm{CD}_{n}$, at the lower $\phi=0.60$. However, we find Newtonian rheology and the overdamped limit at the denser $\phi=0.82$, where behavior becomes very similar to that of $\mathrm{CD}$. Thus, in contrast to $\mathrm{CD}$, where we only find a transition from Newtonian to Bagnoldian rheology at large $Q$ where inertial effects become important, for $\mathrm{CD}_{t}$ we see such a transition as $\phi$ increases even in the limit of $Q \rightarrow 0$, i.e., $m_{s} \rightarrow 0$.

To help understand the origin of the different rheologies, we note that for the collisional models $\mathrm{CD}, \mathrm{CD}_{n}$, and $\mathrm{CD}_{t}$ the overdamped limit is associated with the formation of large clusters of particles (for RD see the Supplemental Material [24]). When the majority of particles cohere together into clusters, particle accelerations become negligible and, hence, the kinetic term in the equation of motion can be neglected. This connection is shown in Fig. 2 where we plot the average particle contact number $z$ vs $\phi$,
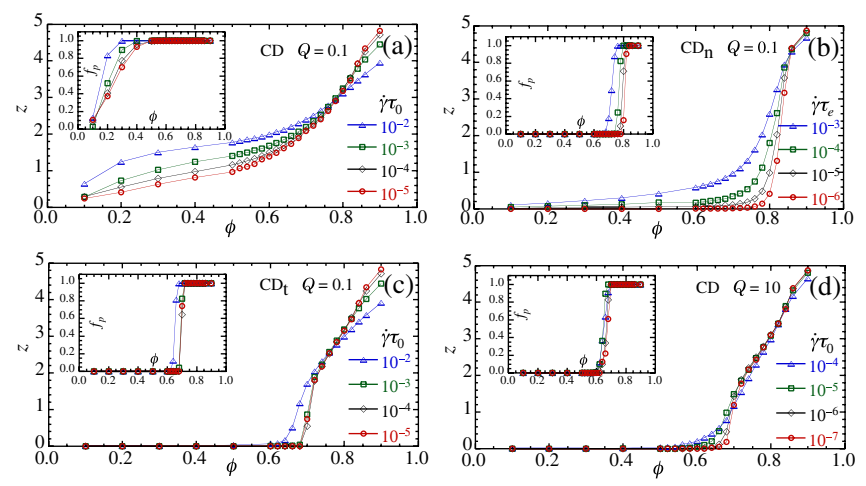

FIG. 2 (color online). Average contact number $z$ vs $\phi$ at different strain rates $\dot{\gamma}$ for models (a) $\mathrm{CD}$, (b) $\mathrm{CD}_{n}$, and (c) $\mathrm{CD}_{t}$ at $Q=0.1$ and (d) $\mathrm{CD}$ at $Q=10$. Insets show the fraction of states $f_{p}$ with percolating connected clusters. for different values of the applied strain rate $\dot{\gamma}$. The inset to each figure shows the fraction of states $f_{p}$ that contain a percolating connected cluster of particles [25].

Figures 2(a)-2(c) are for strongly inelastic collisions, $Q=0.1$. In Fig. 2(a) for model CD, where the rheology is overdamped, $z$ stays finite down to low $\phi$; the percolation fraction remains $f_{p}>0$ down to similarly low $\phi$. The reason for this is simple. For such strongly inelastic collisions, the velocity difference of two colliding particles decays to zero during the collision, and the particles remain in contact. In Fig. 2(b) for model $\mathrm{CD}_{n}$, however, where the rheology is in the inertial limit, $z$ and $f_{p}$ drop rapidly to zero as $\phi$ decreases below $\phi_{J} \approx 0.843$; the drop sharpens as $\dot{\gamma}$ decreases, suggesting that $z, f_{p} \rightarrow 0$ for all $\phi<\phi_{J}$ as $\dot{\gamma} \rightarrow 0$. Again, the reason is simple. Although the normal component of the velocity difference decays to zero during a collision, the tangential component remains finite and causes the particles to move apart, breaking contact. In Fig. 2(c) we show model $\mathrm{CD}_{t}$. Here, we see that $z$ and $f_{p}$ remain finite as $\phi$ decreases below $\phi_{J}$, but they drop sharply to zero at $\phi^{*} \approx 0.7$; this marks the transition from the inertial limit at $\phi<\phi^{*}$ to the overdamped limit at $\phi>\phi^{*}$, as seen in Figs. 1(g) and 1(h). Finally, in Fig. 2(d) we show model CD again, but now for the case of large $Q=10$ where inertial effects are important. We see a transition, with $z$ and $f_{p}$ dropping sharply to zero just below $\phi \approx 0.7$, marking the transition from Bagnoldian rheology at low $\phi$ to Newtonian rheology at high $\phi$, as seen in Figs. 1(c) and $1(\mathrm{~d})$.

We can qualitatively explain the observed transitions as follows. When the system is dilute, particles separate whenever the total velocity difference of the colliding particles is not damped to zero during the collision. This occurs for model $\mathrm{CD}_{t}\left(\mathrm{CD}_{n}\right)$ even in the strongly inelastic limit of small $Q$, since the normal (tangential) component of the velocity difference does not get damped at all. For $\mathrm{CD}$, it happens only at larger $Q$ values when collisions are less inelastic. For dense systems, however, many body effects become important. At sufficiently dense $\phi$, normally directed relative particle motion becomes energetically prohibitive; it is a compressive motion that would induce particle overlaps, and so is constrained by the dense particle geometry. This is in contrast to tangential relative particle motion that corresponds to a local shear deformation with particles sliding around each other with minimal overlaps. Thus, in $\mathrm{CD}_{n}$, where tangential relative motion is not damped, particles continue to separate after collisions. But in $\mathrm{CD}_{t}$ and $\mathrm{CD}$, where tangential relative motion is damped, particles form clusters. Indeed, we find that for all models, at densities $0.6 \lesssim \phi$ the relative motion of particles in contact is almost always tangential, with the particles' separation $\mathbf{r}_{i}-\mathbf{r}_{j}$ very nearly orthogonal to their relative motion $\mathbf{v}_{i}-\mathbf{v}_{j}$ (see the Supplemental Material [24]). A similar result was found for the response of statically jammed packings to a small shear deformation [26]. 
To conclude, we have shown that the rheology of soft-core frictionless disks is strongly dependent on the specific form of the dissipative interaction. At dense $\phi$ in collisional models, tangential dissipation is crucial for the particle clustering that gives Newtonian rheology. Bagnoldian rheology results when particles separate after collisions and the average contact number $z \rightarrow 0$. Sharp transitions between Bagnoldian and Newtonian rheology may exist as a function of particle density $\phi$ and the degree of inelasticity of collisions as measured by $Q$. In the small $Q$ (small $m_{s}$ ) regime of strongly inelastic collisions, the rheology curves approach a limiting form in both the overdamped and inertial cases that extends from the low $\dot{\gamma}$ hard-core limit into the higher $\dot{\gamma}$ region where soft-core effects are manifest.

This work was supported by NSF Grant No. DMR1205800 and Swedish Research Council Grant No. 20103725. Simulations were performed on resources provided by the Swedish National Infrastructure for Computing (SNIC) at PDC and HPC2 N. We thank B. Tighe, H. Hayakawa, and C. Maloney for helpful discussions.

[1] A. J. Liu and S. R. Nagel, Nature (London) 396, 21 (1998).

[2] A. J. Liu, S. R. Nagel, W. van Saarloos, and M. Wyart, in Dynamical Heterogeneities in Glasses, Colloids, and Granular Media, edited by L. Berthier, G. Biroli, J.-P. Bouchaud, L. Cipeletti, and W. van Saarloos (Oxford University Press, New York, 2010).

[3] M. van Hecke, J. Phys. Condens. Matter 22, 033101 (2010).

[4] P. Olsson and S. Teitel, Phys. Rev. Lett. 99, 178001 (2007).

[5] T. Hatano, M. Otsuki, and S. Sasa, J. Phys. Soc. Jpn. 76, 023001 (2007).

[6] R. Lespiat, S. Cohen-Addad, and R. Höhler, Phys. Rev. Lett. 106148302 (2011).

[7] F. Boyer, E. Guazzelli, and O. Pouliquen, Phys. Rev. Lett. 107, 188301 (2011).

[8] R. A. Bagnold, Proc. R. Soc. A 225, 49 (1954).

[9] F. da Cruz, S. Emam, M. Prochnow, J.-N. Roux, and F. Chevoir, Phys. Rev. E 72, 021309 (2005).
[10] G. Lois, A. Lemaître, and J. M. Carlson, Phys. Rev. E 72, 051303 (2005).

[11] M. Otsuki and H. Hayakawa, Phys. Rev. E 80, 011308 (2009).

[12] D. J. Durian, Phys. Rev. Lett. 75, 4780 (1995); Phys. Rev. E 55, 1739 (1997).

[13] G. Katgert, A. Latka, M. E. Möbius, and M. van Hecke, Phys. Rev. E 79, 066318 (2009).

[14] C. Cassar, M. Nicolas, and O. Pouliquen, Phys. Fluids 17, 103301 (2005).

[15] J. Schäfer, S. Dippel, and D. E. Wolf, J. Phys. I France 6, 5 (1996).

[16] S. Tewari, D. Schiemann, D. J. Durian, C. M. Knobler, S. A. Langer, and A. J. Liu, Phys. Rev. E 60, 4385 (1999).

[17] F. Varnik, S. Mandal, V. Chikkadi, D. Denisov, P. Olsson, D. Vågberg, D. Raabe, and P. Schall, Phys. Rev. E 89, 040301(R) (2014).

[18] D. J. Evans and G. P. Morriss, Statistical Mechanics of Non-equilibrium Liquids (Academic, London, 1990).

[19] A. Lemaître, J.-N. Roux, and F. Chevoir, Rheol. Acta 48, 925 (2009).

[20] C. S. O'Hern, L. E. Silbert, A. J. Liu, and S. R. Nagel, Phys. Rev. E 68, 011306 (2003).

[21] B. Andreotti, J.-L. Barrat, and C. Heussinger, Phys. Rev. Lett. 109, 105901 (2012); see Fig. 2 in preprint version, arXiv:1112.1194v1.

[22] D. Vågberg, P. Olsson, and S. Teitel, arXiv:1312.5158.

[23] A. Fall, A. Lemaître, F. Bertrand, D. Bonn, and G. Ovarlez, Phys. Rev. Lett. 105, 268303 (2010); M. Trulsson, B. Andreotti, and P. Claudin, Phys. Rev. Lett. 109, 118305 (2012). Here, the authors find a crossover from Newtonian to Bagnoldian behavior at fixed $\phi$ as $\dot{\gamma}$ increases in a system that is roughly a combination of our $\mathrm{CD}$ and $\mathrm{RD}$. We, however, refer to transitions in rheology in the $\dot{\gamma} \rightarrow 0$ limit.

[24] See Supplemental Material at http://link.aps.org/ supplemental/10.1103/PhysRevLett.112.208303 for further details.

[25] T. Shen, C. S. O'Hern, and M. D. Shattuck, Phys. Rev. E 85, 011308 (2012). Here, the authors find a percolation transition in compression-driven jamming that is at a $\phi$ value well below what we find here.

[26] W. G. Ellenbroek, M. van Hecke, and W. van Saarloos, Phys. Rev. E 80, 061307 (2009). 